\title{
Seasonal study on Bothriocephalus as indicator of metal pollution in yellowfish, South Africa
}

\author{
N-R Retief ${ }^{1}$, A Avenant-Oldewage $^{1 *}$ and $\mathrm{HH}$ du Preez ${ }^{1,2}$ \\ ${ }^{1}$ Department of Zoology, University of Johannesburg, PO Box 524, Auckland Park, 2006, South Africa \\ ${ }^{1}$ Department of Zoology, University of Johannesburg, PO Box 524, Auckland Park, 2006, South Africa \\ ${ }^{2}$ Analytical Services, Hydrobiology Section, Barrage Road, PO Box 3526, Vereeniging, South Africa
}

\begin{abstract}
Eighty largemouth yellowfish, Labeobarbus kimberleyensis, were collected between April 2005 and February 2006 with gill nets close to the island $\left(26^{\circ} 52,249^{\prime} \mathrm{S}, 28^{\circ} 10,249^{\prime} \mathrm{E}\right)$ in the Vaal Dam. The fish were killed, weighed and their length determined. Muscle, liver and spinal cord tissues were collected from each fish and the intestines removed and opened to expose Bothriocephalus acheilognathi. The tapeworms were collected in glass bottles and frozen. Water and sediment, as well as liver, muscle and tapeworm samples were digested and thereafter metal concentrations of 23 elements (lithium, beryllium, titanium, vanadium, chromium, manganese, iron, cobalt, nickel, copper, zinc, arsenic, selenium, molybdenum, cadmium, tin, antimony, tellurium, barium, mercury, thallium, lead and uranium) were determined with an ICP-MS. Bioconcentration of metals (selenium, mercury, and lead during autumn; copper, zinc, selenium, cadmium, antimony, thallium and mercury during winter; lithium, zinc, selenium, cadmium and antimony during spring; and zinc during summer) occurred in tapeworms. The highest mean value was recorded in sediment, followed by water, tapeworms and host tissue. A seasonal trend showed that a higher concentration of the metals had accumulated in tapeworms during winter when water levels were at their lowest.
\end{abstract}

Keywords: Bothriocephalus acheilognathi, bioaccumulation, microwave digestion, ICP-MS, sediment, water

\section{Introduction}

Metals are present at low concentrations in natural aquatic ecosystems, and an increase in the levels of available metals may lead to an increase in the accumulation of the metals in organs and tissues of organisms. Metal bioaccumulation occurs with many toxic pollutants and will concentrate at very high levels in organisms from very low levels in water (Mance, 1987). Accumulation of pollutants depends on external and internal factors in organisms. Bioindicators are used because they are good sentinel species that tend to be ubiquitous, sedentary and long-lived, and have a high pollution tolerance and the ability to accumulate large quantities of a toxin. Temperature and salinity (Philips, 1980) also affect the rate of metal accumulation.

In Europe, interest in parasites in bioaccumulation studies has increased due to parasites' potential as indicators of environmental quality. Recently it was shown that acanthocephalans and cestodes of fish accumulate heavy metals to levels which are magnitudes greater than those in the host itself (Sures, 2004; Petrlova et al., 2007; Jirsa et al., 2008). The majority of the investigations have examined the effects of various forms of pollution on the abundance and distribution of parasites, and the combined effects of pollution and parasitism on the well-being of the hosts (Mackenzie et al., 1995; Lafferty, 1997; Sures et al., 1999a; b; Zimmermann et al.,

\footnotetext{
* To whom all correspondence should be addressed.

正 +2711 559 2449; fax: +2711 559 2286;

e-mail: goldewage@uj.ac.za

Received 1 August 2008; accepted in revised form 19 March 2009.
}

2004; Sanchez-Ramirez et al., 2007). Numerous researchers have investigated Cestoda and Acanthocephala as indicators in pollution studies (Jirsa et al., 2008). Adult acanthocephalans accumulate extremely high burdens of heavy metals and may be used as sensitive bioindicators in monitoring heavy metals. Other reports explain that heavy metals reached higher concentrations in Cestoda than in Acanthocephala, but not for the metal lead (Turčeková and Hanzelová, 1997). The metal concentrations in adult acanthocephalans will respond quickly to changes in environmental exposure of their hosts (Sures et al., 1999a; b; Singer et al., 2005; Sures et al., 2007).

Bioaccumulation studies on fishes in South African rivers have been done in the Limpopo, Crocodile and Vaal Rivers. However, it was only recently indicated that intestinal parasites in the Vaal Dam have potential as indicators of metal pollution (Retief et al., 2006). In a study by Retief et al. (2006) the tapeworms had a higher mean value of metal concentrations than the host tissue in 8 (lithium, beryllium, manganese, selenium, mercury, thallium, lead and uranium) of the 23 elements studied and the second highest mean value of metal concentrations in 7 (chromium, iron, zinc, molybdenum, cadmium, tin and barium) of the 23 elements studied. It was suggested that future studies concentrate on the effect of season, based on this observation. This study was built on the previous one but explored the effect of seasonality on the concentration of metals. This is important due to the fact that the Vaal River is situated in a summer rainfall area and is characterised by varying water levels that influence the metal concentration in the environment. The data from the 2006 study were included and used as the summer survey - to compare it with the data from the other 3 seasons. This is the first reported study of the effect of season on the accumulation of metals in intestinal parasites. 


\section{Materials and methods}

\section{Tissue collection}

Twenty largemouth yellowfish, Labeobarbus kimberleyensis (Gilchrist and Thompson, 1913), were collected with gill nets during each season (April 2005 to February 2006). They were collected in the Vaal Dam close to the island $\left(26^{\circ} 52.249^{\prime} \mathrm{S}\right.$, $\left.28^{\circ} 10.249^{\prime} \mathrm{E}\right)$. The Vaal Dam is the third largest impoundment by volume in South Africa and supplies drinking water to approximately $10 \mathrm{~m}$. people. It was constructed in 1938 and has a capacity of $2.57 \mathrm{bn} . \mathrm{m}^{3}$, a surface area of $300 \mathrm{~km}^{2}$ and a shoreline of over $800 \mathrm{~km}$ and is situated in a catchment area covering $39000 \mathrm{~km}^{3}$

The fish were killed by severing the spinal cord, weighed and the length of each fish recorded. Liver, muscle and vertebrae tissues were dissected and placed in $25 \mathrm{~m} \ell$ glass bottles, while intestines were removed and placed in Petri dishes containing saline solution. These intestines were opened with Dumont tweezers to expose the tapeworms, which were then carefully removed and stored in $25 \mathrm{ml}$ glass bottles. All the tissue samples were frozen until metal analysis. An Ekman Grab was used to collect a sediment sample and a water sample was collected in a $1 \ell$ bottle during each survey.

\section{Microwave digestion}

Tissue samples (liver, muscle, spinal cord and tapeworms) were defrosted and a $1 \mathrm{~g}$ sub-sample was weighed. Individual tissue samples (liver, muscle and tapeworms) were placed in microwave digestion flasks with $5 \mathrm{~m} \ell$ Suprapur ${ }^{\circledR}$ nitric acid $65 \%$ and $1 \mathrm{~m} \ell$ Milli-Q water (18.2 $\Omega$ ) and digested. Spinal cord tissue was digested with $2.5 \mathrm{~m} \ell$ Suprapur ${ }^{\circledR}$ nitric acid $65 \%, 2.5 \mathrm{~m} \ell$ hydrochloric ${ }^{\circledR}$ acid and $1 \mathrm{~m} \ell$ Milli-Q water. Water samples were prepared by filtering the water through $0.45 \mu \mathrm{m}$ acid-membrane filters, and $5 \mathrm{~m} \ell$ Suprapur ${ }^{\circledR}$ nitric acid $65 \%$ was added. Sediment samples $(1 \mathrm{~g})$ were digested as described for liver, muscle and tapeworms. A tissue sub-sample was dried in an oven at $100^{\circ} \mathrm{C}$ to calculate the moisture content in the different tissues.

\section{ICP-MS analysis}

An ICP-MS was used to determine the metal concentrations in each tissue type. Samples were transferred into $15 \mathrm{~m} \ell$ Falcon tubes and the internal standard solution Indium was added to each sample for metal analysis. The concentration of antimony, arsenic, barium, beryllium, cadmium, chromium, cobalt, copper, iron, lead, lithium, manganese, mercury, molybdenum, nickel, selenium, tellurium, thallium, tin, titanium, uranium, vanadium and zinc was determined.

\section{Dry weight determination}

The metal concentrations were determined as $\mu \mathrm{g} / \ell$, then converted to $\mu \mathrm{g} / \mathrm{g}$ to calculate the wet tissue weight and lastly transformed to $\mu \mathrm{g} / \mathrm{m} \ell$ to determine the dry mass incorporating the percentage moisture value.

\section{Statistical analysis}

SPSS for Windows 13.0 (SPSS Inc.) was used for statistical analysis of the data. One-sample t-tests were used to determine whether water and sediment values were significantly different from host tissues and tapeworms. ANOVA was used to determine significant differences between fish tissues and tapeworms seasonally. The Huynh-Feldt test was used to identify significant differences, while Levene's test for equality of error variances was used to determine significant differences and to show which post hoc test should be used to interpret the data.

\section{Results}

All the largemouth yellowfish were infected with $B$. acheilognathi, with an average of $120 \pm 88$ tapeworms per intestine.

In Table 1 metal concentrations in water $(\mu \ell / \ell)$ and sediment $(\mu \mathrm{g} / \mathrm{g})$ are presented. The highest sediment and water concentrations were recorded in the winter survey.

In Table 2 it is shown that the highest mean value for metals occurred in sediment and water. Tapeworms bioaccumulated

\begin{tabular}{|c|c|c|c|c|c|c|c|c|}
\hline & ediment & $\mu g / g)$ an & $\begin{array}{l}\text { vater ( } \\
\text { al Dam }\end{array}$ & $\begin{array}{l}\text { TABLE } 1 \\
\text { /e) mean } \\
\text { ecorded }\end{array}$ & $\begin{array}{l}\text { netal c } \\
\text { easona }\end{array}$ & centra & is for th & \\
\hline & & Sed & ient & & & & & \\
\hline Element & Autumn & Winter & Spring & Summer & Autumn & Winter & Spring & Summer \\
\hline $\mathbf{L i}$ & 0.118 & 0.2923 & 0.9697 & 1.233 & 0.083 & 0.11 & 0 & 3.16 \\
\hline $\mathrm{Be}$ & 0.0368 & 0.0574 & 0.0406 & 0.0023 & 0 & 0.014 & 0.03 & 0.03 \\
\hline $\mathrm{Ti}$ & 28.4393 & 55.0677 & 56.4741 & 54.3911 & 2.17 & 5.51 & 9.76 & 0.26 \\
\hline V & 4.372 & 7.1452 & 15.3918 & 12.6034 & 1.457 & 1.628 & 2.02 & 0.76 \\
\hline $\mathrm{Cr}$ & 33.003 & 34.6387 & 45.5244 & 41.118 & 1.976 & 5.367 & 0 & 0 \\
\hline Mn & 70.4052 & 15.4326 & 26.8053 & 21.0448 & 3.151 & 34.34 & 37.14 & 5.92 \\
\hline $\mathrm{Fe}$ & 2076.23 & 3305.926 & 3972.69 & 3469.708 & 60.53 & 523.7 & 0 & 0 \\
\hline Co & 0.0812 & 0.1269 & 1.4614 & 1.1146 & 0.04 & 0.37 & 1.99 & 0.04 \\
\hline $\mathrm{Ni}$ & 0.8223 & 1.5357 & 2.2173 & 2.5454 & 2.993 & 2.899 & 27.16 & 8.36 \\
\hline $\mathrm{Cu}$ & 1.3173 & 2.0642 & 5.3032 & 11.0748 & 3.74 & 2.777 & 1.67 & 5.39 \\
\hline Zn & 2.6003 & 3.9985 & 10.5609 & 12.9889 & 14.94 & 13.01 & 0 & 11.1 \\
\hline As & 0.2122 & 0.3226 & 0.5087 & 0.3934 & ד.676 & $\begin{array}{l}10.01 \\
0.417\end{array}$ & 0.5 & 0.5 \\
\hline Se & 0.553 & 1.0949 & 0.0881 & 0.1001 & 2.907 & 3.735 & 0 & 0 \\
\hline Mo & 0 & 0 & 0.1052 & 0.0051 & 2.098 & 0 & 4.14 & 0.27 \\
\hline Cd & 0.2931 & 0.037 & 0 & 0 & 0.002 & 0 & 0 & 0 \\
\hline Sn & 0.0116 & 0.0257 & 0.1967 & 0.1796 & 0.163 & 0.255 & 0.67 & 0.32 \\
\hline Sb & 0 & 0 & 0 & 0 & 0.016 & 0 & 0 & 0 \\
\hline Te & $2.00 \mathrm{E}-04$ & $3.00 \mathrm{E}-04$ & 0 & 0 & 0 & 0 & 0 & 0 \\
\hline Ba & 6.4124 & 10.0699 & 8.9108 & 9.9448 & 21.12 & 19.39 & 0 & 0 \\
\hline $\mathrm{Hg}$ & 0.0014 & 0.9465 & 0 & 0 & 0.513 & 0.125 & 0 & 0 \\
\hline Tl & 0.0014 & 0.0038 & 0.025 & 0.0177 & 0.001 & 0.001 & 0 & 0 \\
\hline Pb & 0.6625 & 1.7807 & 2.6982 & 2.2103 & 0.754 & 0.985 & 0.09 & 0.04 \\
\hline $\mathbf{U}$ & 0.0158 & 0.0273 & 0.1097 & 0.1135 & 0.035 & 0.014 & 0.09 & 0 \\
\hline
\end{tabular}




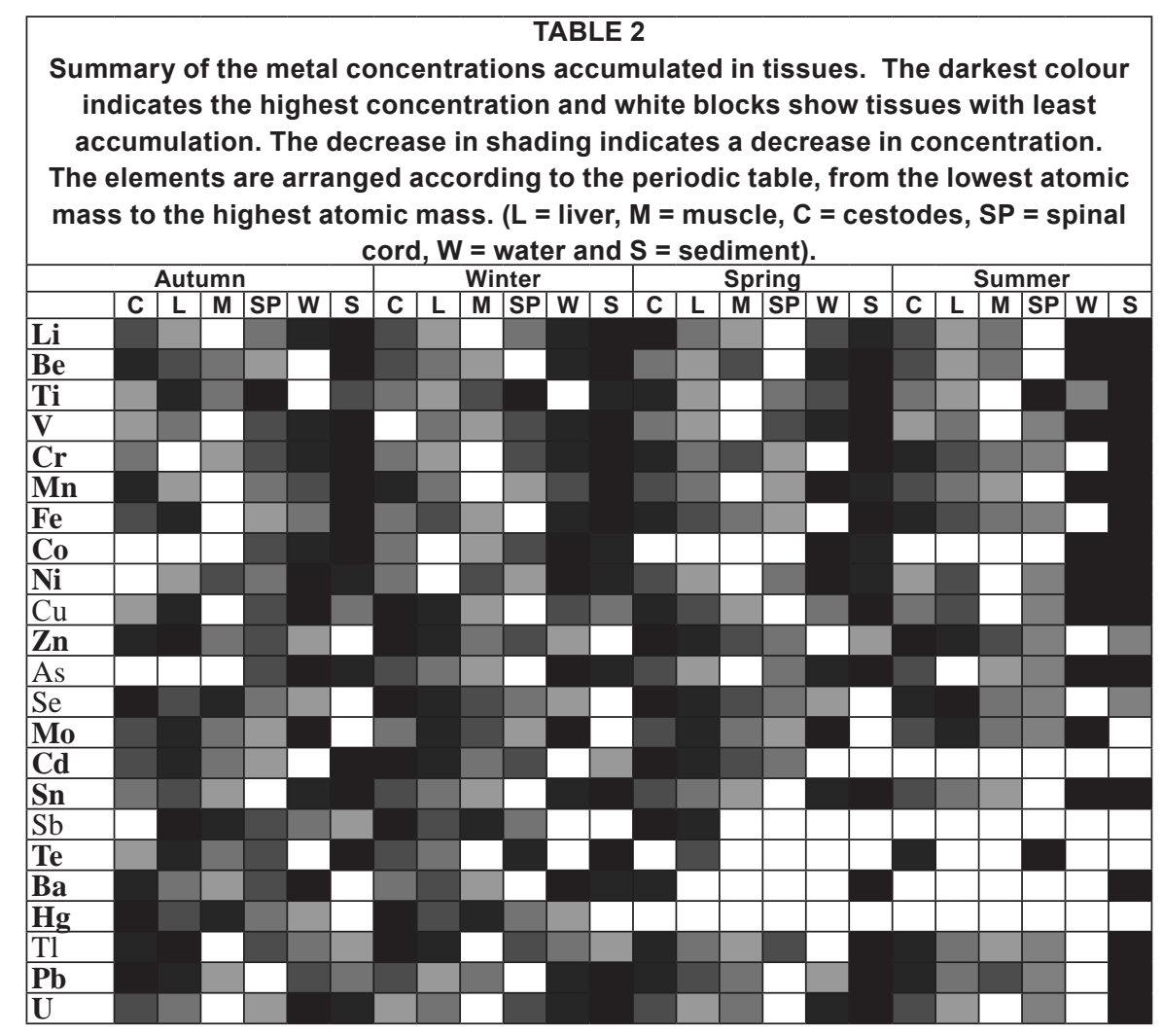

metals (selenium, mercury and lead in autumn; copper, zinc, selenium, cadmium, antimony, thallium and mercury in winter; lithium, zinc, selenium, cadmium and antimony in spring, and zinc in summer) to orders of magnitude higher than those in host tissues, but the following trend type was observed for almost all of the elements: sediment $>$ water $>$ tapeworms $>$ liver $>$ spinal cord $>$ muscle.

Statistical analysis was performed with ANOVA to compare seasonal differences for tissue data (Table 3). When the repeated measures test for lithium (Li) was used to analyse the data, no sphericity could be assumed by Mauchly's test of sphericity. The Huynh-Feld test showed significant differences $(P=0.24)$ seasonally between tissue groups for lithium and significant differences $(P=0.47)$ were recorded when seasons were compared. When the repeated measures test for beryllium (Be) was used to analyse the data, no sphericity could be assumed by Mauchly's test of sphericity, and the Huynh-Feld test showed significant differences $(\mathrm{P}=0.84)$ between tissue groups seasonally for beryllium, while significant differences $(P=0.21)$ were recorded when seasons were compared. When the repeated measures test for titanium (Ti) was used to analyse the data, no sphericity could be assumed by Mauchly's test of sphericity, and the Huynh-Feld test showed no significant differences $(\mathrm{P}<0.05)$ between tissue groups and seasons. When paired-sample t-tests were used to analyse the data, no significant differences were observed between spinal cord and tapeworms $(\mathrm{P}<0.05)$. No significant differences were seasonally recorded, when the Levene's test of equality was performed, while the Dunnett T3 test revealed significant differences between spring-summer $(\mathrm{P}=$ $0.53)$ and summer-spring $(\mathrm{P}=0.53)$. When the repeated measures test for vanadium (V) was used to analyse the data, no sphericity could be assumed by Mauchly's test of sphericity, and the Huynh-Feld test showed no significant differences $(\mathrm{P}<0.05)$ between tissue groups and seasons. When paired-sample t-tests were used to analyse the data, significant differences were observed in muscle and tapeworms $(P=0.39)$, while the Dunnett T3 test showed significant differences between winter-autumn $(\mathrm{P}=1.00)$, spring-summer $(\mathrm{P}=0.52)$ and summer-spring $(\mathrm{P}=$ 0.522). When the repeated measures test for chromium (Cr) was used to analyse the data, no sphericity could be assumed by Mauchly's test of sphericity, and the Huynh-Feld test showed significant differences between tissue groups $(\mathrm{P}=0.13)$ and seasons $(\mathrm{P}=0.06)$. When the repeated measures test for manganese (Mn) was used to analyse the data, no sphericity could be assumed by Mauchly's test of sphericity, and the Huynh-Feld test showed significant differences between tissue groups ( $\mathrm{P}=$ $0.16)$ and seasons $(P=0.32)$. When the repeated measures test for iron (Fe) was used to analyse the data, no sphericity could be assumed by Mauchly's test of sphericity, and the Huynh-Feld test showed no significant differences $(\mathrm{P}<0.05)$ between tissue groups and seasons. When paired-sample t-tests were used to analyse the data significant differences were observed in liver and tapeworms $(\mathrm{P}=0.57)$, while the Dunnett T3 test showed no significant differences between autumn-summer $(\mathrm{P}<0.05)$, winter-summer $(\mathrm{P}<0.05)$, summer-autumn $(\mathrm{P}<0.05)$ and summer-winter $(\mathrm{P}<0.05)$. When the repeated measures test for cobalt (Co) was used to analyse the data, no sphericity could be assumed by Mauchly's test of sphericity, and the Huynh-Feld test showed no significant differences $(\mathrm{P}<0.05)$ between tissue groups and seasons. When paired-sample t-tests were used to analyse the data, no significant differences $(P<0.05)$ were recorded between fish tissues and tapeworms, while the Dunnett T3 test showed no significant differences between seasons $(\mathrm{P}<$ 0.05). When the repeated measures test for nickel (Ni) was used to analyse the data, no sphericity could be assumed by Mauchly's test of sphericity, and the Huynh-Feld test showed significant differences between tissue groups $(\mathrm{P}=0.61)$ and seasons $(\mathrm{P}$ $=0.33)$. When the repeated measures test for copper $(\mathbf{C u})$ was used to analyse the data, no sphericity could be assumed by Mauchly's test of sphericity, and the Huynh-Feld test showed no 


\begin{tabular}{|c|c|c|c|c|c|c|c|c|c|c|c|c|c|c|c|c|}
\hline \multicolumn{17}{|c|}{$\begin{array}{c}\text { TABLE } 3 \\
\text { Descriptive statistics obtained by ANOVA to compare fish tissues and tapeworms seasonally. } \\
\text { The means in } \mu \mathrm{g} / \mathrm{g} \text { are provided between tissue groups. }\end{array}$} \\
\hline $\begin{array}{l}\text { Mean } \\
\text { tissue }\end{array}$ & \multicolumn{4}{|c|}{ Tape-worms } & \multicolumn{4}{|c|}{ Liver } & \multicolumn{4}{|c|}{ Muscle } & \multicolumn{4}{|c|}{ Spinal Cord } \\
\hline Season & Autumn & Winter & Spring & Summer & Autumn & Winter & Spring & Summer & Autumn & Winter & Spring & Summer & Autumn & Winter & Spring & Summer \\
\hline $\mathrm{Li}$ & 1.2 & 0.03 & 1.44 & 0.28 & 0.001 & 0.007 & 0.074 & 0.015 & 0.001 & 0.004 & 0.02 & 0.07 & 0.006 & 0.007 & 0.003 & 0.006 \\
\hline Be & 0.01 & 0.06 & 0.001 & 0.14 & 0.005 & 0.005 & 0.0007 & 0.001 & 0.002 & 0.003 & 0.002 & 0.001 & 0.001 & 0.002 & 0 & \begin{tabular}{|l|}
0.0009 \\
\end{tabular} \\
\hline Ti & 16.66 & 31 & 10.17 & 0.35 & 35.15 & 30.69 & 0.74 & 0.46 & 17.71 & 42.44 & 0.58 & 0.58 & 96.43 & 232.93 & 3.95 & 2.65 \\
\hline $\mathbf{V}$ & 0 & 0.06 & 0.06 & 0 & 0.11 & 0.35 & 0.03 & 0.02 & 0 & 0.06 & 0 & 0 & 0.77 & 0.39 & 0.07 & 0.24 \\
\hline $\mathrm{Cr}$ & 0.42 & 0.31 & 6.28 & 0.14 & 0 & 0.14 & 0.11 & 0.11 & 0.41 & 0.39 & 0.31 & 0.11 & 1.42 & 0.46 & 0.14 & 0.06 \\
\hline Mn & 5.21 & 36.55 & 3.26 & 0.26 & 1.55 & 2.62 & 0.13 & 0.09 & 0.16 & 0.57 & 0.04 & 0.06 & 1.46 & 1.21 & 0 & 0.04 \\
\hline $\mathrm{Fe}$ & 401 & 154.4 & 188.9 & 18.4 & 534.4 & 341 & 11.7 & 9.2 & 54.83 & 50.92 & 6.23 & 3.67 & 67.8 & 32.03 & 0.61 & 1.13 \\
\hline Co & 0 & 0.03 & 0 & 0 & 0 & 0.01 & 0 & 0 & 0 & 0.15 & 0 & 0 & 0.03 & 0.1 & 0 & 0 \\
\hline $\mathrm{Ni}$ & 0.01 & & 0.46 & 0.001 & 0.08 & & 0.04 & 0.04 & 0.29 & 5 & 0.02 & 0.0006 & & & 0.06 & 0.03 \\
\hline Cu & 0.26 & 6 & 6.2 & , & & 11 & 0.82 & 0.67 & 0 & 4 & 0. & 0.03 & & & 0 & 2 \\
\hline Zn & 24.1 & 101.87 & 153.25 & 31.61 & 60.75 & 32.71 & 10.82 & 4.61 & 3.14 & 10.52 & 4.4 & 4.5 & 23.3 & 22.54 & 1.52 & 9 \\
\hline As & 0 & 0.25 & 0.05 & 0.04 & 0 & 0.12 & 0.05 & 0.02 & 0 & 0.11 & 0.002 & 0.02 & .01 & 0.11 & 0.02 & .02 \\
\hline Se & 31.87 & 13 & 1.09 & 0.03 & 16.91 & 5.65 & 0.17 & 0.09 & 19.56 & 4.7 & 0.03 & 0.03 & 7.61 & 2.32 & 0.009 & 0.01 \\
\hline Mo & 0.35 & 0.16 & 0 & 0 & 1.07 & 1.41 & 0.03 & 0.02 & 0.26 & 0.38 & \begin{tabular}{|l|}
$1 \mathrm{E}-05$ \\
\end{tabular} & 0 & 0.04 & 0.11 & 0 & 0 \\
\hline Cd & 0.05 & 0.22 & 0.009 & $1 \mathrm{E}-05$ & 0.1 & 0.12 & 0.001 & 0.0001 & 0.18 & 0.004 & \begin{tabular}{|l|}
0.0003 \\
\end{tabular} & 9E-05 & 0.01 & 0.03 & 5E-05 & $3 \mathrm{E}-05$ \\
\hline Sn & 0.05 & 0.13 & 0.04 & 0.01 & 0.07 & 0.08 & 0.01 & 0.005 & 0.31 & 0.02 & \begin{tabular}{|l|}
0.002 \\
\end{tabular} & \begin{tabular}{|l|}
0.002 \\
\end{tabular} & 0.02 & 0.005 & 0 & \begin{tabular}{|l|}
0.0006 \\
\end{tabular} \\
\hline Sb & 0 & 1.66 & 0.009 & 0 & 0.59 & 0.43 & \begin{tabular}{|l|}
0.0002 \\
\end{tabular} & 0.0005 & 0.08 & 0.6 & $1 \mathrm{E}-05$ & 0 & 0.02 & 0.24 & 0.0003 & 0 \\
\hline Te & 0.00009 & 0.0004 & 0.0004 & 0 & $1 \mathrm{E}-04$ & 0.00009 & 0.0001 & 0 & 3E-05 & 0 & 0 & 0 & $3 \mathrm{E}-05$ & \begin{tabular}{|l|}
0.0002 \\
\end{tabular} & 0 & 1E-05 \\
\hline Ba & 8.69 & 3.01 & 0.18 & 0.002 & 2.57 & 6.08 & 0 & 0.001 & 0.82 & 0.98 & 0.0002 & 0.45 & 3.29 & 13.99 & 0.22 & 4.44 \\
\hline $\mathrm{Hg}$ & 10.4 & 8.74 & 0.02 & 0.004 & 3.32 & 0.59 & 0.007 & 0.004 & 5.91 & 0.6 & 0.01 & 0.01 & 1.6 & 0.32 & 0.004 & 0.005 \\
\hline Tl & 0.002 & 0.003 & 0.001 & $2 \mathrm{E}-04$ & 0.002 & 0.002 & $1 \mathrm{E}-05$ & $1 \mathrm{E}-05$ & 0.0001 & 0.002 & 0 & 3E-06 & 0.0005 & \begin{tabular}{|l|}
0.0001 \\
\end{tabular} & 0.0007 & 0.0001 \\
\hline $\mathbf{P b}$ & 3.29 & 0.44 & 0.91 & 0.19 & 1.07 & 0.25 & 0.05 & 0.02 & 0.2 & 0.07 & 0.05 & 0.03 & 0.15 & 0.04 & 0 & 0.007 \\
\hline $\mathbf{U}$ & 007 & 0.0002 & 0.02 & $7 E-04$ & 0.002 & 0.0004 & 0.0003 & 0.0001 & 0.0002 & 0 & 0.0004 & 0.0001 & 0.002 & 0.0009 & 0 & 0.0002 \\
\hline $\begin{array}{l}\text { Std dev } \\
\text { tissue } \\
\text { type }\end{array}$ & \multicolumn{4}{|c|}{ Tapeworms } & \multicolumn{4}{|c|}{ Liver } & \multicolumn{4}{|c|}{ Muscle } & \multicolumn{4}{|c|}{ Spinal cord } \\
\hline Season & Autumn & Winter & Spring & Summer & Autumn & Winter & Spring & Summer & Autumn & Winter & Spring & Summer & Autumn & Winter & Spring & Summer \\
\hline $\mathrm{Li}$ & & & 6.32 & 1.05 & 0.005 & 0.02 & 0.1 & 0.03 & 0.004 & 0.008 & 0.05 & 0.3 & 0.02 & 0.005 & 0.01 & 0.007 \\
\hline Be & & & 0.018 & 0.036 & & & 0.001 & 0.002 & 0.004 & 0.002 & 0.002 & 0.002 & & 0.002 & 0.0001 & 0 \\
\hline Ti & 6.94 & 10.85 & 31.51 & 0.22 & .16 & & 1.15 & 0.09 & 16.27 & 10.35 & 0.11 & 0.14 & 86.42 & \begin{tabular}{|l|}
140.55 \\
\end{tabular} & 5.23 & 0.62 \\
\hline V & 0 & 06 & 0.26 & 0 & 0.23 & & 0.05 & 0.01 & 0 & 0.06 & 0 & 0 & 0.71 & 0.23 & 0.07 & 2 \\
\hline $\mathrm{Cr}$ & & & 17.44 & .27 & 0 & & 0.18 & 0.14 & 121 & 1 & 0 & 0.04 & & & 0.08 & 3 \\
\hline Mn & 11.08 & 133.38 & 9.85 & 0.42 & 2.95 & & 0.16 & 0.03 & 0.42 & 0.42 & 0.02 & 0.04 & 1.22 & 1.16 & 0 & 0.06 \\
\hline Fe & 810.1 & 195.8 & 567.9 & 30.4 & 413.3 & 165.4 & 11.6 & 3.05 & 117.4 & 111.5 & 1.14 & 0.82 & 71 & 20.7 & 0.54 & 3.53 \\
\hline Co & 0 & 0.06 & 0 & 0 & 0 & 0.004 & 0 & 0 & 0 & 0.06 & 0 & 0 & 0.03 & 0.05 & 0 & 0 \\
\hline $\mathrm{Ni}$ & 0.03 & 0.95 & 1.63 & 0.005 & 0.36 & 0.73 & 0.09 & 0.13 & 1.24 & 1.37 & 0.02 & 0.002 & 0.3 & 0.77 & 0.03 & 0.02 \\
\hline Cu & 1.17 & 14.9 & 18.7 & 0.09 & 9.52 & 4.3 & 1.05 & 0.34 & 0 & 0.86 & 0.015 & 0.02 & 5.35 & 0.35 & 0 & 0.22 \\
\hline Zn & 35.93 & 120.47 & 449.96 & 48.73 & 108.9 & 10.3 & 17.76 & 1.12 & 6.88 & 5.14 & 0.66 & 0.88 & 19.2 & 8.73 & 1.54 & 0.45 \\
\hline As & 0 & 0.23 & 0.15 & 0.11 & 0 & 0.08 & 0.005 & 0.002 & 0 & 0.07 & 0.002 & 0.02 & 0.63 & 0.16 & 0.03 & 0.01 \\
\hline Se & 39.6 & 34.2 & 3.59 & 0.05 & 11.13 & 5.03 & 0.23 & 0.04 & 14.76 & 3.87 & 0.02 & 0.02 & 6.85 & 2.69 & 0.002 & 0.009 \\
\hline Mo & 0.82 & 0.33 & 0 & 0 & 0.95 & 1.33 & 0.05 & 0.008 & 0.6 & 0.9 & 0.0006 & 0 & 0.04 & 0.21 & 0 & 0 \\
\hline Cd & 0.06 & 0.57 & 0.02 & 0.00007 & 0.1 & 0.16 & 0.002 & 0.0007 & 0.04 & 0.01 & 0.0004 & 0.0003 & 0.02 & 0.03 & 0.0002 & 0.02 \\
\hline Sn & 0.12 & 0.33 & 0.1 & 0.03 & 0.07 & 0.13 & 0.02 & 0.02 & 0.08 & 0.027 & 0.001 & 0.003 & 0.03 & 0.006 & 0 & 0.002 \\
\hline Sb & 0 & 6.52 & 0.03 & 0 & 1.06 & 0.53 & 0.0007 & \begin{tabular}{|l|}
0.00002 \\
\end{tabular} & 0.21 & 2.16 & 0.00005 & 0 & 0.03 & 0.07 & 0.0009 & 0 \\
\hline Te & 0.00003 & 0.00008 & 0.002 & 0 & 0.0003 & 0.00004 & \begin{tabular}{|l|}
0.0005 \\
\end{tabular} & 0 & 0.00007 & 0 & 0 & 0 & 0.00005 & 0.0005 & 0 & 0.00006 \\
\hline Ba & 16.6 & 4.6 & 0.77 & 0.006 & 6.64 & 23.95 & 0 & 0.004 & 2.5 & 0.91 & 0.0005 & 0.004 & 4.82 & 7.77 & 0.41 & 0.08 \\
\hline $\mathrm{Hg}$ & 18.5 & & 0.08 & 0.01 & 3.74 & & 0.01 & 0.007 & 5.91 & 0.6 & \begin{tabular}{|c|}
0.01 \\
\end{tabular} & 0.01 & 2.63 & 0.2 & 0.005 & 0.003 \\
\hline Tl & 0.002 & 0.002 & 0.004 & 0.0004 & 0.003 & 0.001 & 0.00005 & 0.00004 & $\begin{array}{c}0.0007 \\
\end{array}$ & 0.0004 & 0 & 0.00001 & 0.0008 & 0.0006 & 0.0009 & 0.0001 \\
\hline Pb & 9.21 & 0.99 & 2.52 & 0.34 & 3.38 & 0.54 & 0.08 & 0.009 & 0.59 & 0.11 & 0.02 & 0.008 & 0.39 & 0.8 & 0 & 0.03 \\
\hline $\mathbf{U}$ & 0.03 & 0.0003 & 0.06 & 0.001 & 0.005 & 0.0007 & 0.0003 & 0.00008 & 0.00008 & 0 & 0.0005 & 0.0001 & 0.004 & 0.0007 & 0 & 0.002 \\
\hline
\end{tabular}

significant differences $(\mathrm{P}<0.05)$ between tissue groups and seasons. When paired-sample t-tests were used to analyse the data, significant differences were observed in liver-tapeworms ( $\mathrm{P}=$ $0.43)$, while the Dunnett T3 test showed significant differences between autumn-spring $(\mathrm{P}=1.00)$, autumn-summer $(\mathrm{P}=0.05)$, spring-autumn $(\mathrm{P}=1.00)$, spring-summer $(\mathrm{P}=0.64)$, summerautumn $(P=0.05)$ and summer-spring $(P=0.64)$. When the repeated measures test for zinc (Zn) was used to analyse the data, no sphericity could be assumed by Mauchly's test of sphericity, and the Huynh-Feld test showed significant differences $(\mathrm{P}<0.05)$ for tissue groups, while significant differences were recorded for seasons $(\mathrm{P}=0.17)$. When paired-sample $\mathrm{t}$-tests were used to analyse the data, no significant differences were observed in muscle-tapeworms $(\mathrm{P}<0.05)$. When the repeated measures test for arsenic (As) was used to analyse the data, no sphericity could be assumed by Mauchly's test of sphericity, and the Huynh-Feld test showed no significant differences $(\mathrm{P}<0.05)$ for tissue groups, while significant differences were recorded between seasons $(\mathrm{P}=0.05)$. When paired-sample t-tests were used to analyse the data no significant differences were observed in liver-tapeworms $(\mathrm{P}<0.05)$, muscle-tapeworms $(\mathrm{P}<0.05)$ and spinal cord-tapeworms $(\mathrm{P}<0.05)$. When the repeated measures test for selenium (Se) was used to analyse the data, no sphericity could be assumed by Mauchly's test of sphericity, and the Huynh-Feld test showed no significant differences $(\mathrm{P}<0.05)$ for tissue groups, while significant differences were observed between seasons $(\mathrm{P}=0.06)$. When paired-sample t-tests were used to analyse the data, no significant differences were observed in spinal tapeworms $(\mathrm{P}<0.05)$. When the repeated measures test for molybdenum (Mo) was used to analyse the data, no sphericity could be assumed by Mauchly's test of sphericity, and the Huynh-Feld test showed no significant differences $(\mathrm{P}<0.05)$ for tissue groups and seasons $(\mathrm{P}<0.05)$. When paired-sample t-tests were used to analyse the data no significant differences were observed in liver and tapeworms $(\mathrm{P}<0.05)$, while the Dunnett $\mathrm{T} 3$ test showed significant differences between 
TABLE 4

Summary of the statistical comparisons (1-sample t-test) of the metal levels in the water (W) compared to the liver (L), muscle (M), tapeworms (T) and spinal cord (SC). * = values cannot be calculated because the standard deviation was 0 .

\begin{tabular}{|c|c|c|c|c|c|c|c|c|c|c|c|c|c|c|c|c|c|c|c|c|c|c|c|c|}
\hline Season & $\begin{array}{l}\text { Tissue } \\
\text { types }\end{array}$ & Li & $\mathrm{Be}$ & Ti & V & $\mathrm{Cr}$ & $\mathrm{Mn}$ & $\mathrm{Fe}$ & Co & $\mathrm{Ni}$ & $\mathrm{Cu}$ & $\mathrm{Zn}$ & As & Se & Mo & Cd & Sn & Sb & Te & $\mathrm{Ba}$ & $\mathrm{Hg}$ & TI & $\mathrm{Pb}$ & U \\
\hline \multirow{4}{*}{ 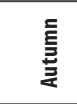 } & $W \Leftrightarrow T$ & 0.05 & $>0.05$ & $>0.05$ & * & 0.05 & $>0.05$ & $>0.05$ & * & $<0.05$ & $<0.05$ & $>0.05$ & $*$ & $<0.05$ & $<0.05$ & $<0.05$ & $<0.05$ & * & $>0.05$ & $<0.05$ & $>0.05$ & 0.05 & 0.05 & 0 \\
\hline & $\mathbf{W} \Leftrightarrow \mathbf{L}$ & $<0.05$ & $>0.05$ & $>0.05$ & $<0$ & * & $>0.05$ & $<0.05$ & * & $<0.05$ & $>0.05$ & $>0.05$ & * & $<0.05$ & $<0.05$ & $<0.05$ & $<0.05$ & $>0.05$ & $>0.05$ & $<0.05$ & $<0.05$ & $>0.05$ & $>0.05$ & $<0.05$ \\
\hline & $\mathbf{W} \Leftrightarrow \mathbf{M}$ & $<0.05$ & $>0.05$ & $<0.05$ & * & $<0.05$ & $<0.05$ & $>0.05$ & * & $<0.05$ & * & $<0.05$ & * & $<0.05$ & $<0.05$ & $>0.05$ & $>0.05$ & $>0.05$ & $>0.05$ & $<0.05$ & $>0.05$ & $<0.05$ & $<0.05$ & $<0.05$ \\
\hline & $\mathrm{W} \Leftrightarrow \mathrm{SC}$ & 0.05 & $>0.05$ & $<0.05$ & 0.05 & $>0.05$ & $<0.05$ & $>0.05$ & 0.05 & $<0.05$ & $>0.05$ & $>0.05$ & 0.05 & $<0.05$ & $<0.05$ & $<0.05$ & $<0.05$ & $>0.05$ & $>0.05$ & $<0.05$ & $<0.05$ & $>0.05$ & $<0.05$ & $<0.05$ \\
\hline \multirow{4}{*}{ 竎 } & $\Leftrightarrow \mathbf{T}$ & $<0.05$ & $>0.05$ & $<0.05$ & $<0.05$ & $<0.05$ & $>0.05$ & $<0.05$ & $<0.05$ & $<0.05$ & $<0.05$ & $<0.05$ & $<0.05$ & $>0.05$ & $>0.05$ & $>0.05$ & $>0.05$ & $>0.05$ & $>0.05$ & $<0$ & $>0.05$ & $<0$ & $>0.05$ & $<0.05$ \\
\hline & $\mathbf{W} \Leftrightarrow \mathbf{L}$ & $<0.05$ & $<0.05$ & $<0.05$ & $<0.05$ & $<0.05$ & $<0.05$ & $<0.05$ & $<0.05$ & $<0.05$ & $<0.05$ & $<0.05$ & $<0.05$ & $>0.05$ & $<0.05$ & $<0.05$ & $<0.05$ & $<0.05$ & $>0.05$ & $>0.05$ & $>0.05$ & $<0.05$ & $<0.05$ & $<0.05$ \\
\hline & $\mathbf{W} \Leftrightarrow \mathbf{M}$ & $<0.05$ & $<0.05$ & $<0.05$ & $<0.05$ & $<0.05$ & $<0.05$ & $<0.05$ & $<0.05$ & $<0.05$ & $<0.05$ & $>0.05$ & $<0.05$ & $>0.05$ & $>0.05$ & $>0.05$ & $>0.05$ & $>0.05$ & \begin{tabular}{|l|}
$*$ \\
\end{tabular} & & $<0$ & $<0.05$ & & * \\
\hline & SC & $<0.05$ & $<0.05$ & $<0.05$ & $<<0.05$ & $<0.05$ & $<0.05$ & $<0.05$ & $<0.05$ & $<0.05$ & $<0.05$ & $<0.05$ & $<0.05$ & $>0.05$ & $<0.05$ & $<0.05$ & $<0.05$ & $>0.05$ & $>0.05$ & $<0$ & $<0.05$ & $<0.05$ & \begin{tabular}{l|}
$<<0.05$ \\
\end{tabular} & $<0.05$ \\
\hline \multirow{4}{*}{ 高 } & $\mathrm{W} \Leftrightarrow \mathbf{T}$ & $>0.05$ & $<0.05$ & $>0.05$ & $<0.05$ & $>0.05$ & $<0.05$ & $>0.05$ & * & $<0.05$ & $>0.05$ & $>0.05$ & $<0.05$ & $>0.05$ & * & $>0.05$ & $<0.05$ & $>0.05$ & $>0.05$ & $>0.05$ & $>0.05$ & $>0.05$ & $>0.05$ & $<0.05$ \\
\hline & $\mathbf{W} \Leftrightarrow \mathbf{L}$ & $<0.05$ & $<0.05$ & $<0.05$ & $<0$ & $<0.05$ & $<0.05$ & $<0.05$ & . & $<0.05$ & $<0.05$ & $>0.05$ & $<0.05$ & $<0.05$ & $<0.05$ & $>0.05$ & $<0.05$ & $>0.05$ & $>0.05$ & * & $>0.05$ & $>0.05$ & $>0.05$ & $<0.05$ \\
\hline & $\mathbf{W} \Leftrightarrow \mathbf{M}$ & $>0.05$ & $<0.0$ & $<0.05$ & * & $<0.05$ & $<0.05$ & $<0.05$ & F & $<0.05$ & $<0.05$ & $<0.05$ & $<0.05$ & $<0.05$ & $<0.05$ & $>0.05$ & $<0.05$ & $>0.05$ & 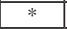 & $>0.05$ & $<0.05$ & - & $<0.05$ & $<0.05$ \\
\hline & $\mathrm{W} \Leftrightarrow \mathrm{SC}$ & $>0.05$ & $<0.05$ & $<0.05$ & $<0$ & $<0.05$ & * & $<0.05$ & $*$ & $<0.05$ & * & $<0.05$ & $<0.05$ & $>0.05$ & * & $>0.05$ & 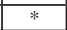 & $>0.05$ & * & $>0.05$ & $<0$. & $<0.05$ & * & * \\
\hline \multirow{4}{*}{ 离 } & $W \Leftrightarrow T$ & $<0.05$ & $<0.05$ & $>0.05$ & * & $>0.05$ & $<0.05$ & $>0.05$ & . & $<0.05$ & $<0.05$ & $>0.05$ & $<0.05$ & $>0.05$ & * & $>0.05$ & $<0.05$ & * & & $>0.05$ & $>0.05$ & $>0.05$ & $>0.05$ & $>0.05$ \\
\hline & $\mathbf{W} \Leftrightarrow \mathbf{L}$ & $<0.05$ & $<0.05$ & $<0.05$ & $<0.05$ & $<0.05$ & $<0.05$ & $<0.05$ & * & $<0.05$ & $<0.05$ & $<0.05$ & $<0.05$ & $<0.05$ & $<0.05$ & $>0.05$ & $<0.05$ & $>0.05$ & * & $>0.05$ & $>0.05$ & $>0.05$ & $<0.05$ & $<0.05$ \\
\hline & $\mathbf{W} \Leftrightarrow \mathbf{M}$ & $<0.05$ & $<0.05$ & $<0.05$ & * & $<0.05$ & $<0.05$ & $<0.05$ & & $<0.05$ & $<0.05$ & $<0.05$ & $<0.05$ & $<0.05$ & 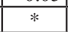 & $>0.05$ & 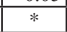 & & & $>0.05$ & $<0.05$ & $>0.05$ & $<0.05$ & $<0.05$ \\
\hline & $V \Leftrightarrow S C$ & $<0.05$ & 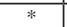 & $<0.05$ & $\mid<0.05$ & $<0.05$ & $<0.05$ & $>0.05$ & 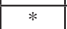 & $<0.05$ & $<0.05$ & $<0.05$ & $<0.05$ & $<0.05$ & & $>0.05$ & $<0.05$ & * & $>0.05$ & $<0.05$ & $<0.05$ & $<0.05$ & $<0.05$ & $>0.0$ \\
\hline
\end{tabular}

TABLE 5

Summary of the statistical comparisons (1-sample t-test) of the metal levels in the sediment (S) compared to the liver (L), muscle (M), tapeworms (T) and spinal cord (SC). ${ }^{*}=$ values cannot be calculated because the standard deviation was 0 .

\begin{tabular}{|c|c|c|c|c|c|c|c|c|c|c|c|c|c|c|c|c|c|c|c|c|}
\hline Season & $\begin{array}{l}\text { Tissue } \\
\text { types }\end{array}$ & Li & Be & $\mathrm{Ti}$ & V & $\mathrm{Cr}$ & $\mathrm{Fe}$ & Co & $\mathrm{Ni}$ & As & Se & Mo & Cd & Sn & Te & $\mathrm{Ba}$ & $\mathrm{Hg}$ & TI & $\mathrm{Pb}$ & U \\
\hline \multirow{4}{*}{ 衰 } & $\mathbf{S} \Leftrightarrow \mathbf{T}$ & $<0.05$ & $<0.05$ & $>0.05$ & * & $<0.05$ & $\begin{array}{c}<0.05 \\
\end{array}$ & * & $<<0.05$ & * & $<0.05$ & $>0.05$ & $<0.05$ & $>0.05$ & $>0.05$ & $>0.05$ & $<0.05$ & $<0.05$ & $>0.05$ & $<0.05$ \\
\hline & $\mathbf{S} \Leftrightarrow \mathbf{L}$ & $<0.05$ & $<0.05$ & $>0.05$ & $<0.05$ & * & $<0.05$ & * & $<0.05$ & * & $<0.05$ & $<0.05$ & $<0.05$ & $<0.05$ & $>0.05$ & $<0.05$ & $<0.05$ & $<0.05$ & $>0.05$ & $<0.05$ \\
\hline & $\mathbf{S} \Leftrightarrow \mathbf{M}$ & $<0.05$ & $<0.05$ & $<0.05$ & * & $<0.05$ & $<0.05$ & * & $>0.05$ & * & $<0.05$ & $>0.05$ & $<0.05$ & $>0.05$ & $>0.05$ & $<0.05$ & $<0.05$ & $<0.05$ & $<0.05$ & $<0.05$ \\
\hline & $\mathrm{S} \Leftrightarrow \mathrm{SC}$ & $<0.05$ & $<0.05$ & $<0.05$ & 0.05 & $<0.05$ & $<0.05$ & 0.05 & $<0.05$ & $<0.05$ & $<0.05$ & $<0.05$ & $<0.05$ & $<0.05$ & $<0.05$ & $<0.05$ & $<0.05$ & $>0.05$ & $<0.05$ & $<0.05$ \\
\hline \multirow{3}{*}{$\stackrel{\text { İ }}{\stackrel{ \pm}{\Sigma}}$} & $\mathbf{S} \Leftrightarrow \mathbf{L}$ & $<0.05$ & $<0.05$ & $<<0.05$ & $<0.05$ & $<0.05$ & $<0.05$ & $<0.05$ & $<0.05$ & $<0.05$ & $<0.05$ & $<0.05$ & $<0.05$ & $>0.05$ & $>0.05$ & $>0.05$ & $<<0.05$ & $<0.05$ & $<0.05$ & $<0.05$ \\
\hline & $\mathbf{S} \Leftrightarrow M$ & $<0.05$ & $<0.05$ & $<0.05$ & $<0.05$ & $<0.05$ & $<0.05$ & $<0.05$ & $<0.05$ & $<0.05$ & $<0.05$ & $>0.05$ & $<0.05$ & $>0.05$ & * & $<0.05$ & $>0.05$ & $<0.05$ & $<0.05$ & * \\
\hline & $\mathbf{S} \Leftrightarrow \mathbf{S C}$ & $<0.05$ & $<0.05$ & $<0.05$ & $<0.05$ & $<0.05$ & $<0.05$ & $<0.05$ & $<0.05$ & $<0.05$ & $>0.05$ & $>0.05$ & $<0.05$ & $<0.05$ & $>0.05$ & $<0.05$ & $<0.05$ & $<0.05$ & $<0.05$ & $<0.05$ \\
\hline \multirow{4}{*}{ 离 } & $\mathbf{S} \Leftrightarrow \mathbf{T}$ & $>0.05$ & $<0.05$ & \begin{tabular}{l|l|}
$<<0.05$ \\
\end{tabular} & $<0.05$ & $<0.05$ & $<0.05$ & * & $<0.05$ & $<0.05$ & $>0.05$ & $*$ & $>0.05$ & \begin{tabular}{l|l|}
$<0.05$ \\
\end{tabular} & $>0.05$ & $<0.05$ & $>0.05$ & $<0.05$ & $<0.05$ & $<0.05$ \\
\hline & $\mathbf{S} \Leftrightarrow \mathbf{L}$ & $<0.05$ & $<0.05$ & $<0.05$ & $<0.05$ & $<0.05$ & $<0.05$ & * & $<0.05$ & $<0.05$ & $>0.05$ & $<0.05$ & $<0.05$ & $<0.05$ & $>0.05$ & * & $<0.05$ & $<0.05$ & $<0.05$ & $<0.05$ \\
\hline & $\mathbf{S} \Leftrightarrow \mathbf{M}$ & $<0.05$ & $<0.05$ & $<0.05$ & * & $<0.05$ & $<0.05$ & * & $<0.05$ & $<0.05$ & $<0.05$ & $<0.05$ & $<0.05$ & $<0.05$ & * & $<0.05$ & $<0.05$ & * & $<0.05$ & $<0.05$ \\
\hline & $\mathbf{S} \Leftrightarrow \mathbf{S C}$ & $<0.05$ & $<0.05$ & $<0.05$ & $<0.05$ & $<0.05$ & $<0.05$ & 7 & $<0.05$ & $<0.05$ & $<0.05$ & $*$ & $>0.05$ & * & * & $<0.05$ & $<0.05$ & $<0.05$ & * & * \\
\hline \multirow{4}{*}{ 离 } & $\mathbf{S} \Leftrightarrow \mathbf{T}$ & $\begin{array}{c}<0.05 \\
\end{array}$ & $>0.05$ & $<0.05$ & * & $2<0.05$ & $\begin{array}{c}<0.05 \\
\end{array}$ & 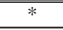 & $\begin{array}{c}<0.05 \\
\end{array}$ & $<0.05$ & $\overline{<<0.05}$ & * & $>0.05$ & $<0.05$ & ** & $\langle<0.05$ & $>0.05$ & $<0.05$ & $\overline{<<0.05}$ & $\begin{array}{ll}<0.05 \\
\end{array}$ \\
\hline & $\mathbf{S} \Leftrightarrow \mathbf{L}$ & $<0.05$ & $<0.05$ & $<0.05$ & $<0.05$ & $<0.05$ & $<0.05$ & * & $<0.05$ & $<0.05$ & $>0.05$ & $<0.05$ & $>0.05$ & $<0.05$ & * & $<0.05$ & $<0.05$ & $<0.05$ & $<0.05$ & $<0.05$ \\
\hline & $\mathbf{S} \Leftrightarrow \mathbf{M}$ & $<0.05$ & $<0.05$ & $<0.05$ & * & $<0.05$ & $<0.05$ & 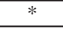 & $<0.05$ & $<0.05$ & $<0.05$ & * & $>0.05$ & $<0.05$ & * & $<0.05$ & $<0.05$ & $<0.05$ & $<0.05$ & $<0.05$ \\
\hline & $\mathrm{S} \Leftrightarrow \mathrm{SC}$ & $<0.05$ & * & \begin{tabular}{c|c|}
$<0.05$ \\
\end{tabular} & $<0.05$ & $<0.05$ & $<0.05$ & * & $<0.05$ & $<0.05$ & $<0.05$ & * & $>0.05$ & \begin{tabular}{l|}
$<0.05$ \\
\end{tabular} & $>0.05$ & $<0.05$ & $<0.05$ & $<0.05$ & $<0.05$ & $<0.05$ \\
\hline
\end{tabular}

spring-summer $(\mathrm{P}=0.88)$ and summer-spring $(\mathrm{P}=0.88)$. When the repeated measures test for cadmium (Cd) was used to analyse the data, no sphericity could be assumed by Mauchly's test of sphericity, and the Huynh-Feld test showed significant differences between tissue groups $(\mathrm{P}=0.06)$ and seasons $(\mathrm{P}=0.10)$. When the repeated measures test for tin (Sn) was used to analyse the data, no sphericity could be assumed by Mauchly's test of sphericity, and the Huynh-Feld test showed no significant differences between tissue groups $(\mathrm{P}<0.05)$, while significant differences were recorded seasonally $(\mathrm{P}=0.40)$. When pairedsample t-tests were used to analyse the data, no significant differences were observed in muscle-tapeworms $(\mathrm{P}<0.05)$ and spinal cord-tapeworms $(\mathrm{P}<0.05)$. When the repeated measures test for antimony (Sb) were used to analyse the data, no sphericity could be assumed by Mauchly's test of sphericity, and the Huynh-Feld test showed significant differences between tissue groups $(P=0.41)$ and seasons $(P=0.41)$. When the repeated measures test for tellurium (Te) was used to analyse the data, no sphericity could be assumed by Mauchly's test of sphericity, and the Huynh-Feld test showed significant differences between tissue groups $(\mathrm{P}=0.44)$ and seasons $(\mathrm{P}=0.29)$. When the repeated measures test for barium (Ba) was used to analyse the data, no sphericity could be assumed by Mauchly's test of sphericity, and the Huynh-Feld test showed no significant differences between tissue groups $(\mathrm{P}<0.05)$ and seasons $(\mathrm{P}<0.05)$. When pairedsample t-tests were used to analyse the data, no significant differences were observed in muscle and tapeworms $(\mathrm{P}<0.05)$, while the Dunnett T3 test showed significant differences between autumn-winter $(\mathrm{P}=0.75)$, spring-summer $(\mathrm{P}=0.98)$, winter-au- tumn $(\mathrm{P}=0.75)$ and summer-spring $(\mathrm{P}=0.98)$. When the repeated measures test for mercury (Hg) was used to analyse the data, no sphericity could be assumed by Mauchly's test of sphericity, and the Huynh-Feld test showed significant differences between tissue groups ( $\mathrm{P}=0.08)$ and seasons $(\mathrm{P}=0.39)$. When the repeated measures test for thallium ( $\mathbf{T l})$ was used to analyse the data, no sphericity could be assumed by Mauchly's test of sphericity, and the Huynh-Feld test showed no significant differences between tissue groups $(\mathrm{P}<0.05)$ while significant differences were recorded between seasons $(\mathrm{P}=0.08)$. When paired-sample t-tests were used to analyse the data, significant differences were found in liver-tapeworms $(P=0.18)$. When the repeated measures test for lead $\mathbf{( P b )}$ was used to analyse the data, no sphericity could be assumed by Mauchly's test of sphericity, and the Huynh-Feld test showed significant differences between tissue groups $(\mathrm{P}=0.06)$ and seasons $(\mathrm{P}=0.31)$. When the repeated measures test for uranium (U) was used to analyse the data, no sphericity could be assumed by Mauchly's test of sphericity, and the Huynh-Feld test showed significant differences between tissue groups $(\mathrm{P}=0.12)$ and seasons $(\mathrm{P}=$ 0.34).

Significant differences detected between the water and tissue samples irrespective of the season are shown in Table 4. Seasonally higher levels of metal concentrations were recorded in water during the winter and spring, while the lowest values were recorded in summer.

Significant differences detected between the sediment and tissue samples irrespective of the season are shown in Table 5. Sediment naturally had higher metal concentration than tissue 
samples and seasonally sediment had the highest metal concentrations during winter and spring, while the lowest values were recorded in summer.

\section{Discussion}

Fish absorb metals from the surrounding water via their gills and skin, through the food that they eat and from water in the intestine. Heath (1987) suggested 4 routes by which metals can be introduced into organisms. Water and food can be regarded as the 2 main sources of metals to freshwater fish. The gills are important due to their large surface area and the short diffusion distances between water and blood (Stagg and Shuttleworth, 1982; Pärt and Lock, 1983; Lloyd, 1992). As a metal passes through the gills and gastrointestinal tract into the body, it is taken up by blood and transported, bound to proteins especially adapted to metallothioneins, or bound to amino acids (Stagg and Shuttleworth, 1982).

Fish also have various routes for the excretion of harmful chemicals, which include the gills, bile (via the faeces), kidneys and the skin (Heath, 1987). The mechanism of metal excretion involves the liver. Since blood from the intestine passes through the liver before reaching the systemic circulation, the liver can remove toxicants from the blood, biotransform or emit it into the bile and avoid distribution to other body parts (Hofer and Lackner, 1995). The bile enters the intestine, where it mixes with the food that is being digested (Heath, 1987). Retief et al. (2007) showed B. acheilognathi attach in the first $10 \%$ of the digestive system's length and they are therefore continuously exposed to bile secretions.

Due to low rainfall in winter, geographical weathering of rock will leach natural elements into the Vaal Dam increasing the metal levels of the system (Chutter, 1963). During this study the highest mean value of metal accumulation was recorded in winter followed by autumn, spring and lastly summer (Tables 3 to 5). This trend was recorded when sediment, water and tapeworms were compared and is not specific to tissue type. The metal concentration trend observed in most of the elements was: sediment $>$ water $>$ tapeworms $>$ liver $>$ spinal cord $>$ muscle (Tables 3 to 5). During the winter months parasite numbers increase (Retief et al., 2007) as immune response in infected fish decreases (Granath and Esch, 1983) because of the reduction in metabolic rate of the host. It is also the time when the level of uptake of metals is lower, yet in this study the concentrations are highest in all tissues and in the tapeworms. The metal concentration in the tissues and the tapeworms mimics the pattern in the water and sediment thereby indicating that the organism accumulates in response to the availability in the environment. This may explain why the tapeworms have accumulated a higher concentration of metals during the winter.

In invertebrates, there are 2 key mechanisms of detoxification: (1) the activation of metal-binding proteins such as metallothioneins (Janssen and Dallinger, 1991); and (2) the compartmentalisation of metals as intracellular granules of different types (Dallinger, 1993). The last mechanism has been described for woodlice (Dallinger and Prosi 1988), diplopods (Köhler et al., 1995), slugs (Howard and Simkiss, 1981) and springtails (Dallinger, 1993). After storage in digestive cells, the metals can be excreted from the body either by direct release of vesicle contents into the lumen of the gut and consequent discharge with the faeces (Dallinger, 1993), or by substitution of the whole mid-gut epithelium (Hubert, 1979) during moulting. Another mechanism employed by tapeworms to excrete metals is via corpuscles (McCullough and Fairweather, 1987; Von Brand et al., 1965; 1967).
In freshwater habitats, invertebrates are bathed in a medium containing dissolved trace metals, and the uptake is passive (Dallinger and Rainbow, 1993). Uptake of major ions like potassium, sodium and calcium requires active pumps to cross the cell membrane. The first mode of uptake depends on the high affinity of trace metals for proteins in the external medium that bind passively with the transport proteins in the membranes of permeable surfaces of the invertebrates. By facilitated diffusion the trace metals are transported across the membrane and into the cell, where they bind with a series of metal-binding ligands of increasing affinity (Dallinger and Rainbow, 1993). Metal uptake continues passively against a concentration gradient.

In aquatic media, dissolved trace metals are partitioned in equilibrium between inorganic and organic complexing ligands. In any unusual circumstances direct passage through the lipid membrane is probably the route of uptake of metals in lipophilic organometallic compounds. Cestodes rely solely on their tegument for food acquisition and the surface area of the mature tapeworm tegument is enlarged up to 10 -fold by finger-like projections. Six separate amino acid carriers have been identified in the rat tapeworm, Hymenolepis diminuta, as well as 3 purine/pyrimidine carriers with multiple-binding capacities (Pappas and Read, 1975). Its free surface bears brush edges of finger-like projections, which are equivalent to microvilli of the host mucosal cells. The delimiting plasma membranes of both structures are rich in carrier molecules that provide a transport facility for sugars, amino acids, etc. Tapeworms have shortened metabolic pathways and only partial oxidation of food stuffs takes place. As a result, they create and excrete great quantities of partially oxidised short-chain organic acids. In adult Taenia solium and Taenia saginata, TGTP1 is expressed in tegument cytons and other cells, possibly for the excretory system (Rodriguez-Contreras et al., 1998).

Sures and Siddall (1999) and Sures (2003) reported that host bile may play an important role in the uptake and accumulation of lead in acanthocephalans. There is a permanent osmotic flow of water over the gills in freshwater fish, and lead ions are able to pass across the epithelial membrane via para-cellular diffusion. The lead ions bind to erythrocytes and are transported via the circulatory system to various organs. The majority of the lead is removed by the liver and excreted into the intestine by the bile. The bile contains steroids with which metal ions form organometallic complexes which pass down the bile duct into the small intestine. These will be reabsorbed by the intestinal wall or run through the hepatic intestinal cycle or be excreted in faeces. Sures and Siddall (1999) and Sures (2003) suggested that organometallic complexes formed in the liver of chub (Leuciscus cephalus) pass down the bile duct into the small intestine and are taken up by the tapeworms with bile salts. This could explain the availability of metals in tapeworms due to their position in close proximity to the bile opening. One reason for the location of tapeworms in close proximity to the bile opening is that tapeworms use the bile salts for their egg formation (Sures and Siddall, 1999). Smyth (1994) also reported that tapeworms have lost the ability to metabolise fatty acids, therefore they use the hosts' metabolites after they have been broken into smaller molecules and this is also a stimulus for egg hatching (Sures et al., 2003).

Taraschewski (2000) suggested that metals in female M. moniliformis are discharged via the shells of their eggs (Sures and Siddall, 1999). The same conclusion has been drawn for B. acheilognathi (Riggs et al., 1987) and B. scorpii (Sures et al., 2007). In both studies the highest metal concentrations were 
recorded in the gravid proglottids when compared to the anterior part of the cestode's strobila, thus a possible storage/elimination in the eggs/cement gland may result in a detoxification of metals. Sures and Siddall (1999) reported that segments of mature tapeworms contained more metals, because these segments had been exposed for longer periods of time to metals than the younger anterior segments. Whole tapeworms of varying sizes were analysed during this study, which could explain the high variance in tapeworm data.

Various authors have reported that $B$. acheilognathi has a short life cycle and completes its life cycle in 1 year (Hoffman, 1976; Mitchell and Hoffman, 1980; Körting, 1975; Paperna, 1996). Due to the short life cycle younger tapeworms and younger segments in older tapeworms would absorb lower concentrations of metals, while mature and gravid proglottids would have been exposed for longer periods and would absorb higher concentrations of metals. Retief et al. (2007) indicated that the mean intensity of tapeworm infection increases from spring to summer, autumn and then winter, indicating that the new cohort is released in spring. During this period the metal concentration is at its lowest. It may be attributed to the lower concentration in the environment but also to the fact that the tapeworms are at their youngest.

Cystacanth larvae of acanthocephala showed only little tendency to accumulate metals, despite the fact that their intermediate host, a crustacean, is in close contact with sediment pollutants (Sures and Taraschewski, 1995). It seems to be a characteristic feature of cestodes to accumulate high volumes of some heavy metals (Sures and Taraschewski, 1995). In the present study it was observed that tapeworms bioaccumulate selenium, mercury and lead in autumn; copper, zinc, selenium, cadmium, antimony, thallium and mercury in winter; whilst lithium, zinc, selenium, cadmium and antimony is absorbed in spring and zinc in summer (Table 2). The disparity in the accumulation capacity of cestodes might be related to specific properties of the tegument, particulates of their life cycles and other complex causes. Due to their accumulation capacity and their abundance in different aquatic ecosystems, cestodes may be useful indicators of metal contamination in aquatic environments in addition to other indicator invertebrates (Sures and Taraschewski, 1995) The findings of the present study corroborate that tapeworms have potential as indicators in pollution studies and that the metal concentration in the worms shows a seasonal variation.

\section{Conclusion}

Significant differences in water and sediment were recorded when fish tissues and tapeworms were compared seasonally and between tissue types. A seasonal trend showed that high metal concentrations were present in the sediment and water in winter, similarly to the tapeworms, which accumulated a higher concentration of metals in the winter. Tapeworms are therefore useful indicators in pollution studies.

\section{Acknowledgements}

We thank Mr Henry Foden for his help and guidance with microwave digestion at the Rand Water Analytical facility and the use of their microwave digester and Mr Francois van Wyk (Rand Water) for providing the water and sediment data. WARFSA (P191) and the University of Johannesburg are thanked for funding and infrastructure.

\section{References}

CHUTTER FM (1963) Hydrobiological studies on the Vaal River in the Vereeniging area, Part I. Introduction: water chemistry and biological studies on the fauna of habitats other than muddy bottom sediments. Hydrobiol. 21 1-65.

DALLINGER R and PROSI F (1988) Heavy metals in the terrestrial isopod Porcellio scaber Latreille. II. Subcellular fractionation of metal-accumulating lysosomes from hepatopancreas. Cell Biol. Toxicol. 4 97-109.

DALLINGER R (1993) Strategies of metal detoxification in terrestrial invertebrates. In: Dallinger R and Rainbow PS (eds.) Ecotoxicology of Metals in Invertebrates. SETAC Special Publication Series. Lewis Publishers, United States of America. 246-289.

DALLINGER R and RAINBOW PS (1993) Ecotoxicology of Metals in Invertebrates. SETAC special Publication Series. Lewis Publishers, United States of America. $461 \mathrm{pp}$.

GRANATH WO and ESCH GW (1983) Temperature and other factors that regulate the composition and infrapopulation densities of Bothriocephalus acheilognathi (Cestoda) in Gambusia affinis (Pisces). J. Parasitol. 69 (6) 1116-1124.

HEATH AG (1987) Water Pollution and Fish Physiology. CRC Press, Inc., Boca Raton,. Florida, United States of America. 245 pp.

HOFER R and LACKNER R (1995) Fischtoxikologie - Theorie und Praxis. Fischer Verlag, Cruston Fisher, Stuttgart/New York. 164 pp.

HOFFMAN GL (1976) The Asian tapeworm, Bothriocephalus gowkongensis, in the United States and research needs in fish parasitology. Proc. 1976 Fish Farming Conference and Annual Convention of the Catfish Farmers of Texas. 2 84-91.

HOWARD B and SIMKISS K (1981) Metal binding by Helix aspersa blood. Comp. Biochem. Physiol. 70 (A) 559-561.

HUBERT M (1979) Localization and identification of mineral elements and nitrogenous waste in Diplopoda. In: Camatini M (ed.) Myriapod Biology. Academic Press Inc., New York. 127-133.

JANSSEN HH and DALLINGER R (1991) Diversification of cadmiumbinding proteins due to different levels of contamination in Arion lusitanicus. Arch. Environ. Contam. Toxicol. 20 132-137.

JIRSA F, DVORAK ML, KRACHLER R and FRANK C (2008) Heavy metals in the Nase, Chondrostoma nasus (L. 1758), and its intestinal parasite Caryophyllaeus laticeps (Pallas 1781) from Austrian rivers: Bioindicative aspects. Arch. Environ. Contam. Toxicol. 55 (4) 619-626.

KÖHLER H-R, KÖRTJE K-H and ALBERTI G (1995) Content, absorption quantities and intracellular storage sites of heavy metals in Diplopoda (Arthropoda). BioMetals 8 37-46.

KÖRTING W (1975) Larval development of Bothriocephalus sp. (Cestoda: Pseudophyllidea) from carp (Cyprinus carpio L.) in Germany. J. Fish Biol. 7 (6) 727-753.

LAFFERTY KD (1997) Environmental parasitology: what can parasites tell us about human impacts on the environment? Parasitol. Today 13 (7) 251-255.

LLOYD R (1992) Pollution and Freshwater Fish. Blackwell Scientific Publications Ltd, Oxford, United Kingdom. 176 pp.

McCULLOUGH JS and FAIRWEATHER I (1987) The structure, composition, formation and possible functions of calcareous corpuscles in Trilocularia acanthiaevulgaris Olsson 1867 (Cestoda, Tetraphyllidea). Parasitol. Resour. 74 175-182.

MACKENZIE K, WILLIAMS HH, WILLIAMS B, MCVICAR AH, and SIDDALL R (1995) Parasites as indicators of water quality and the potential use of helminth transmission in marine pollution studies. Adv. Parasitol. 35 85-144.

MANCE G (1987) Pollution Threat of Heavy Metals in Aquatic Environments. Elsevier Applied Science Publishers Ltd., London, United Kingdom. 372 pp.

MITCHELL AJ and HOFFMAN GL (1980) Important tapeworms of North American freshwater fishes. United States Fish and Wildlife Service Fish Disease Leaflet 59 1-18.

PETRLOVA J, KRISKOVA S, ZITKA O, HUBALEK J, PRUSA R, ADAM V, WANG J, BEKLOVA M, SURES B, KIZEK R (2007) Utilizing a chronopotentiometric sensor technique for metallo- 
thionein determination in fish tissues and their host parasites. Sens. Actuator 127 112-119.

PAPERNA I (1996) Parasites, infections and diseases of fishes in Africa - an update. Food and Agriculture Organisation of the United Nations, Rome. CIFA Technical Paper, No. 31. 220 pp.

PAPPAS PW and READ CP (1975) Membrane transport in helminth parasites - A review. Exp. Parasitol. 33 469-530.

PÄRT P and LOCK RAC (1983) Diffusion of calcium, cadmium and mercury in a mucous solution from rainbow trout. Comp. Biochem. Physiol. 76 C (2) 259-263.

PHILIPS DJH (1980) Quantitative Aquatic Biological Indicators, Their Use to Monitor Trace Metal and Organochlorine Pollution. Applied Science Publishers, London, United Kingdom. 488 pp.

RETIEF N-R, AVENANT-OLDEWAGE A and DU PREEZ HH (2006) The use of cestode parasites from the largemouth yellowfish, Labeobarbus kimberleyensis (Gilchrist and Thompson, 1913) in the Vaal Dam, South Africa, as indicators of heavy metal bioaccumulation. J. Phys. Chem. Earth 31 840-847.

RETIEF N-R, AVENANT-OLDEWAGE A and DU PREEZ HH (2007) Ecological aspects of the occurrence of Asian tapeworm, Bothriocephalus acheilognathi Yamaguti, 1934 infection in the Largemouth yellowfish, Labeobarbus kimberleyensis Gilchrist and Thompson, 1913 in the Vaal Dam, South Africa. J. Phys. Chem. Earth 32 (15-18) 1384-1390.

RIGGS MR, ESCH GW and LEMLY AD (1987) The growth, biomass and fecundity of Bothriocephalus acheilognathi in a North Carolina cooling reservoir. J. Parasitol. 73 (5) 893-900.

RODRIGUEZ-CONTRERAS D, SKELLY PJ, LANDA A, SHOEMAKER CB, and LACLETTE JP (1998) Molecular and functional characterization and tissue localization of 2 glucose transporter homologues (TGTP1 and TGTP2) from the tapeworm Taenia solium. Parasitol. 117 579-588.

SANCHEZ-RAMIREZ C, VIDAL-MARTINEZ VM, AGIRREMACEDO ML, RODRIGUEZ-CANUL RP (2007) Cichlidogyrus sclerosus (Monogenea: Ancyrocephalinae) and its host, the Nile Tilapia (Oreochromis niloticus), as bioindicators of chemical pollution. J. Parasitol. 93 (5) 1097-1106.

SINGER C, ZIMMERMANN S, SURES B (2005) Induction of heat shock proteins (hsp70) in the Zebra mussel (Dreissena polymorpha) following exposure to platinum group metals (platinum, palladium and rhodium): Comparison with lead and chromium exposures. Aquat. Toxicol. 75 65-75.

SMYTH JD (1994) Introduction to Animal Parasitology (3rd edn.). Cambridge University Press, Cambridge, United Kingdom. 549 pp.

STAGG RM and SHUTTLEWORTH TJ (1982) The accumulation of copper in Platichthys flesus L. and its effects on plasma electrolyte concentrations. J. Fish Biol. 20 (4) 491-500.

SURES B (2003) Accumulation of heavy metals by intestinal helminths in fish: facts, appraisal and perspectives. Parasitol. 126 53-60.
SURES B (2004) Environmental parasitology: Relevancy of parasites in monitoring environmental pollution. Trends Parasitol. 20 (4) 170 177.

SURES B and TARASCHEWSKI H (1995) Cadmium concentrations in two adult acanthocephalans, Pomphorynchus laevis and Acanthocephalus lucii, as compared with their fish hosts and cadmium and lead levels in larvae of A. lucii as compared with their crustacean host. Parasitol Res. 81 494-497.

SURES B, TARASCHEWSKI H and ROKICKI J (1997) Lead and cadmium content of two Monobothrium wageneri and Bothriocephalus scorpii, and their fish hosts. Parasitol. Res. 83 618-623.

SURES B and SIDDALL R (1999) Pomphorhynchus laevis: The intestinal acanthocephalan as a lead sink for its host (Leuciscus cephalus). Exp. Parasitol. 93 66-72.

SURES B, SIDDALL R and TARASCHEWSKI H (1999a) Parasites as accumulation indicators of heavy metal pollution. Parasitol. Today 15 16-21.

SURES B, STEINER W, RYDLO M, and TARASCHEWSKI H (1999b) Concentrations of 17 elements in Zebra mussel (Dreissena polymorpha), in different tissues of Perch (Perca fluciatilis), and in perch intestinal parasites (Acanthocephalus lucii) from the subalpin lake Mondsee (Austria). Environ. Toxicol. Chem. 18 (11) 2574-2579.

SURES B, SCHEIBLE T, BASHTAR AR, and TARASCHEWSKI H (2003) Lead concentrations in Hymenolepis diminuta and Taenia taeniaeformis larvae compared to their rat hosts (Rattus norvegicus) sampled from the city of Cairo, Egypt. Parasitol. 127 483-487.

SURES B and ZIMMERMANN S (2007) Impact of humic substances on the aqueous solubility, uptake and bioaccumulation of platinum, palladium and rhodium in exposure studies with Dreissena polymorpha. Environ. Pollut. 146 444-451.

TARASCHEWSKI H (2000) Host-parasite interactions in Acanthocephala: A morphological approach. Adv. Parasit. 46 1-179.

TURČEKOVÁ L and HANZELOVÁ V (1997) Parasites as possible bioindicators of water pollution. 10th Wroclaw Parasitological Conference Karpacz. In: Parasites Sensu Lato in the Modern Laboratories of the World. p. 53. Elzbieta Lonc, University of Wroclaw, Microbiological Institute, Przybyszewskiego str. 63/77, Pl 51-148 Wroclaw, Poland.

VON BRAND T, SCOTT DB, NYLEN MU and PUGH MH (1965) Variations in the mineralogical composition of cestode calcareous corpuscles. Exp. Parasitol. 16 382-391.

VON BRAND T, NYLEN MU, MARTIN GN and CHURCHWELL FK (1967) Composition and crystallization patterns of calcareous corpuscles of cestodes grown in different classes of hosts. J. Parasitol. 53 (4) 683-687.

ZIMMERMANN S, BAUMAN U, TARASCHEWSKI H, SURES B (2004) Accumulation and distribution of platinum and rhodium in the European eel Anguilla anguilla following aqueous exposure to metals. Environ. Pollut. 127 195-202. 